Article

\title{
Identification of BELL Transcription Factors Involved in Nodule Initiation and Development in the Legumes Pisum sativum and Medicago truncatula
}

\author{
Alexandra V. Dolgikh (D), Elizaveta S. Rudaya and Elena A. Dolgikh * \\ All-Russia Research Institute for Agricultural Microbiology, Podbelsky chausse 3, Pushkin, \\ 196608 St. Petersburg, Russia; sqshadol@gmail.com (A.V.D.); rudaya.s.e@gmail.com (E.S.R.) \\ * Correspondence: dol2helen@yahoo.com; Tel.: +7-812-4705100
}

Received: 19 November 2020; Accepted: 17 December 2020; Published: 20 December 2020

\begin{abstract}
Single three-amino acid loop extension (TALE) homeodomain proteins, including the KNOTTED-like (KNOX) and BEL-like (BELL) families in plants, usually work as heterodimeric transcription factor complexes to regulate different developmental processes, often via effects on phytohormonal pathways. Nitrogen-fixing nodule formation in legumes is regulated by different families of homeodomain transcription factors. Whereas the role of KNOX transcription factors in the control of symbiosis was studied early, BELL transcription factors have received less attention. Here, we report the identification and expression analysis of BELL genes in the legume plants Medicago truncatula and Pisum sativum, which are involved in regulating symbiosis initiation and development. A more precise analysis was performed for the most significantly upregulated PsBELL1-2 gene in pea. We found that the PsBELL1-2 transcription factor could be a potential partner of PsKNOX9. In addition, we showed that PsBELL1-2 can interact with the PsDELLA1 (LA) protein-regulator of the gibberellin pathway, which has a previously demonstrated important role in symbiosis development.
\end{abstract}

Keywords: Pisum sativum; Medicago truncatula; BELL transcription factors; genomic and transcriptomic data; phylogenetic tree; gene expression; two hybrid yeast system

\section{Introduction}

The formation of symbiotic nitrogen-fixing nodules represents a process in which the development of new organs substantially depends on external factors [1,2]. In plants, such regulation can be achieved by means of a complex network of transcription factors and phytohormones. However, the exact mechanisms underlying such interactions to regulate nodulation remain unknown.

Three-amino acid loop extension (TALE) homeodomain transcription factors play a comprehensive role in the regulation of numerous biological processes, and members of this superfamily are highly conserved in different organisms [3]. In plants, two families of this superfamily have been discovered, KNOX and BELL. The significant role of TALE proteins in meristem maintenance and morphogenesis regulation has been shown, but specific processes such as chloroplast development, cell wall modification, or leaf formation also represent examples of TALE-regulated processes in plants [4-6].

The special feature of KNOX and BELL transcription factors is their ability to control the expression of genes involved in the regulation of biosynthesis and the degradation of phytohormones, in particular, cytokinins and gibberellins. For example, it has been shown that in the shoot apical meristem, the KNOX transcription factor SHOOT-MERISTEMLESS (STM) in Arabidopsis thaliana can affect the expression of GA20ox and GA2ox genes, which are responsible for the biosynthesis of active forms of gibberellin and their degradation, respectively [7]. It was also found that StBEL5 from Solanum tuberosum forms a 
heterodimeric complex with StPOTH1 (KNOX family), which is extremely important for the regulation of GA20ox expression through the direct binding of this complex to the promoter of the GA20ox gene [8]. It is important to note that KNOX and BELL also have important features such as the ability to move from cell to cell. It has been shown that such movement is achieved through plasmodesmata, and this trafficking is specific [9]. For BELL transcription factors, the long-distance transport ability with phloem flow has been observed wherein it could move from the shoot to the root.

In addition to participating in shoot developmental processes, KNOX and BELL transcription factors also play an important role in root development. It has been shown that members of the KNOX family, such as KNAT6 and BP (KNAT1), influence the development of lateral roots in Arabidopsis, and BLH6 (BELL family) is also involved in this regulation $[8,10,11]$. The role of KNOX transcription factors that belong to class II in the control of new root organ development, specifically the nitrogen-fixing nodules, has been previously examined in several studies. It was demonstrated that the expression of KNOX class II genes is associated with the early stages of nodule development in Medicago truncatula and Pisum sativum L. [12]. The role of MtKNOX3/PsKNOX3 genes in this process has been studied in detail $[13,14]$. It has been found that the KNOX3 transcription factor might influence the expression of cytokinin biosynthesis genes such as IPTs and LOGs during nodulation $[13,14]$.

However, despite the importance of the BELL-KNOX heterodimer complex formation for its functional activity, no data have been presented about the role of BEL-like transcription factors in the control of nodule development. In addition, in contrast to the well-studied mechanism of KNOX and BELL with respect to the effect on gibberellin and cytokinin metabolism in shoots, few data are available about the influence of TALE on phytohormonal processes in roots and nodules. Nevertheless, it has been shown that the exogenous treatment of $S$. tuberosum roots with gibberellins (GA3) is associated with decreased expression of several KNOX genes [9]. Moreover, it was recently shown that the expression levels of several KNOX and BELL genes are essentially decreased in P. sativum mutants, la cry-s (della1 della2), with enhanced sensitivity to gibberellins due to impaired DELLA protein (negative regulators of plant response to these hormones) function [15].

To verify the role of BELL transcription factors in the regulation of symbiosis development in legumes, a genome-wide search of BELL genes was performed in the model legume $M$. truncatula and crop legume pea (P. sativum), and phylogenetic relationships between these genes in legumes and in Arabidopsis thaliana and Oryza sativa were determined. An analysis of transcriptomic data allowed for the identification of BELL genes showing activated expression upon nodulation in M. truncatula. The expression levels of close homologues of these genes in pea were further evaluated using RT-qPCR analysis. This allowed for a more precise analysis of distinct $B E L L$ genes activated during symbiosis development, performed in pea.

\section{Results}

\subsection{Diversity of BEL-Like Genes in Legumes}

To identify BELL genes in the genome of the model legume $M$. truncatula and the crop legume pea P. sativum, we searched for these genes using amino acid sequences of BEL1 and BEL-like genes of $A$. thaliana for BLASTP analysis [16]. As a result, we found 14 genes in the M. truncatula genome sequence v5 [17] and in P. sativum genome sequence v1 [18]. A phylogenetic tree was constructed to assess the homology of the identified genes with $A$. thaliana BELL genes. We found out that only four Arabidopsis genes (AtBEL1, AtBLH5, AtBLH11, and AtATH1) have unique orthologs in $P$. sativum and $M$. truncatula genomes (Figure 1). Based on this tree reconstruction, we determined the phylogenetic relationships among BELL genes in the legumes M. truncatula, P. sativum, Lotus japonicus, Phaseolus vulgaris, and non-legumes A. thaliana, O. sativa (Table S1). 


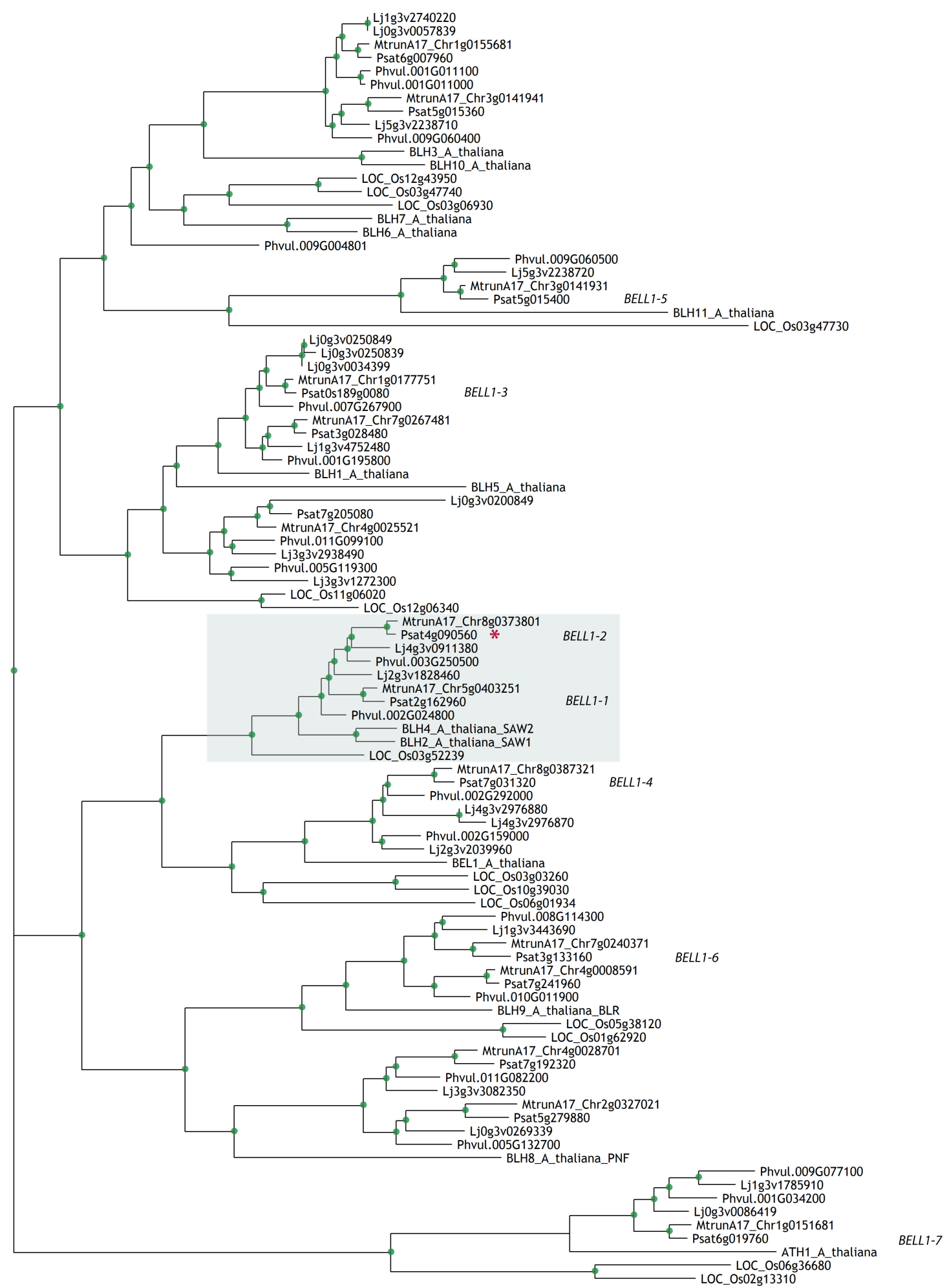

Figure 1. Phylogenetic tree constructed by the maximum-likelihood method based on amino acid sequences of BELLs from Arabidopsis thaliana, rice Oryza sativa and legumes: Medicago truncatula, Pisum sativum, Lotus japonicus, and Phaseolus vulgaris. Green dots indicate support more than 70 based on 1000 bootstrap replicates. Red asterisk indicates the gene of interest.

Most of the available transcriptomic data (RNA-seq) for early stages of symbiosis development in $M$. truncatula were analyzed using the v4 genome sequence as a reference. Since our analysis allowed us to identify $14 B E L L$ genes in M. truncatula genome v5 and only $13 B E L L$ genes were 
found in M. truncatula genome v4, we performed re-analysis of these available transcriptomic data (RNA-seq) using M. truncatula genome sequence v5 as a reference. Data collected at 24 and $48 \mathrm{~h}$ and 3, 4, 5, and 7 days after inoculation (3, 4, 5, and 7 dai) were used for analysis (Figure S1). We found that the expression level of some BELL genes had a similar level in the non-inoculated and inoculated roots; however, for other BELL genes their expression level was significantly increased in response to inoculation (MtBELL1-2, MtBELL1-3, and MtBELL1-4) (MtrunA17_Chr8g0373801, MtrunA17_Chr1g0177751, and MtrunA17_Chr8g0387321, correspondently). For MtBELL1-5 (MtrunA17_Chr3g0141931), strong upregulation (up to 10-fold change) has been shown, although its expression level remained relatively low in inoculated roots.

To evaluate the expression level of homologous BELL genes in P. sativum roots after inoculation, we performed RT-qPCR analysis (Figure 2, Figure S2). The expression dynamics of BELL genes were evaluated at different stages of symbiosis development after inoculation of pea P. sativum with Rhizobium leguminosarum bv. viciae. We found that the expression levels of PsBELL1-2 started to increase at the first stages of symbiosis initiation ( 5 days after inoculation, 5 dai) and reached the maximum level in the nodules (14 dai), as was also shown for homologue of this gene in $M$. truncatula based on transcriptomic analysis (Figure S1). In addition, we detected increased expression of PsBELL1-3 and PsBELL1-4 in pea roots at 7 dai and the highest level of their expression was found in the nodules.

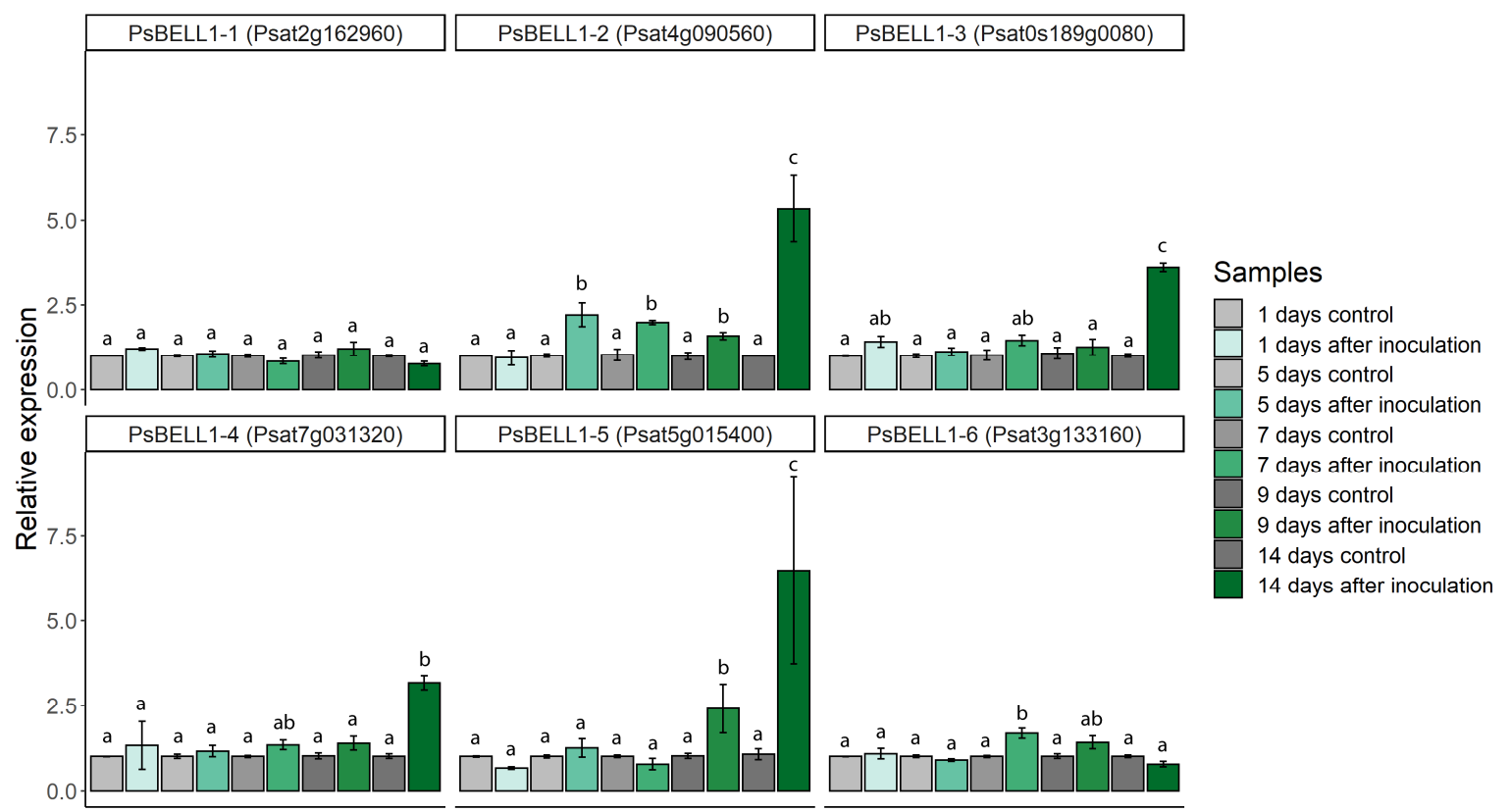

Figure 2. Expression dynamics of selected BELL genes in inoculated Pisum sativum roots (cv. Frisson) at different stages of symbiosis development. mRNA levels were normalized against Ubiquitin and values were calculated as ratios relative to non-inoculated root expression levels (control). The data of three independent biological experiments were analyzed. For one biological experiment, three technical replicates were obtained at each developmental stage. Bars represent the mean \pm SEM. Different letters indicate statistically significant differences between groups as analyzed by one-way analysis of variance (one-way ANOVA), followed by Tukey's post hoc test.

We also found that the expression levels of PsBELL1-2, PsBELL1-3, and PsBELL1-4 were highly increased in the nodules of the other pea line (SGE) (Figure 3). Analysis of these gene expressions in pea nodules based on available RNA-seq data showed similar results (Figure S3). Moreover, these data corresponded to transcriptomic data in M. truncatula nodules [17], where homologues of these genes, MtBELL1-2, MtBELL1-3, and MtBELL1-4, also showed the strongest expression level in the nodules (Figures S4 and S5). Therefore, the expression of MtBELL1-2, MtBELL1-3, and MtBELL1-4 and their putative orthologues PsBELL1-2, PsBELL1-3, and PsBELL1-4 in pea has been shown to be upregulated 
during nodulation in both legumes, suggesting their possible participation in the regulation of this process.

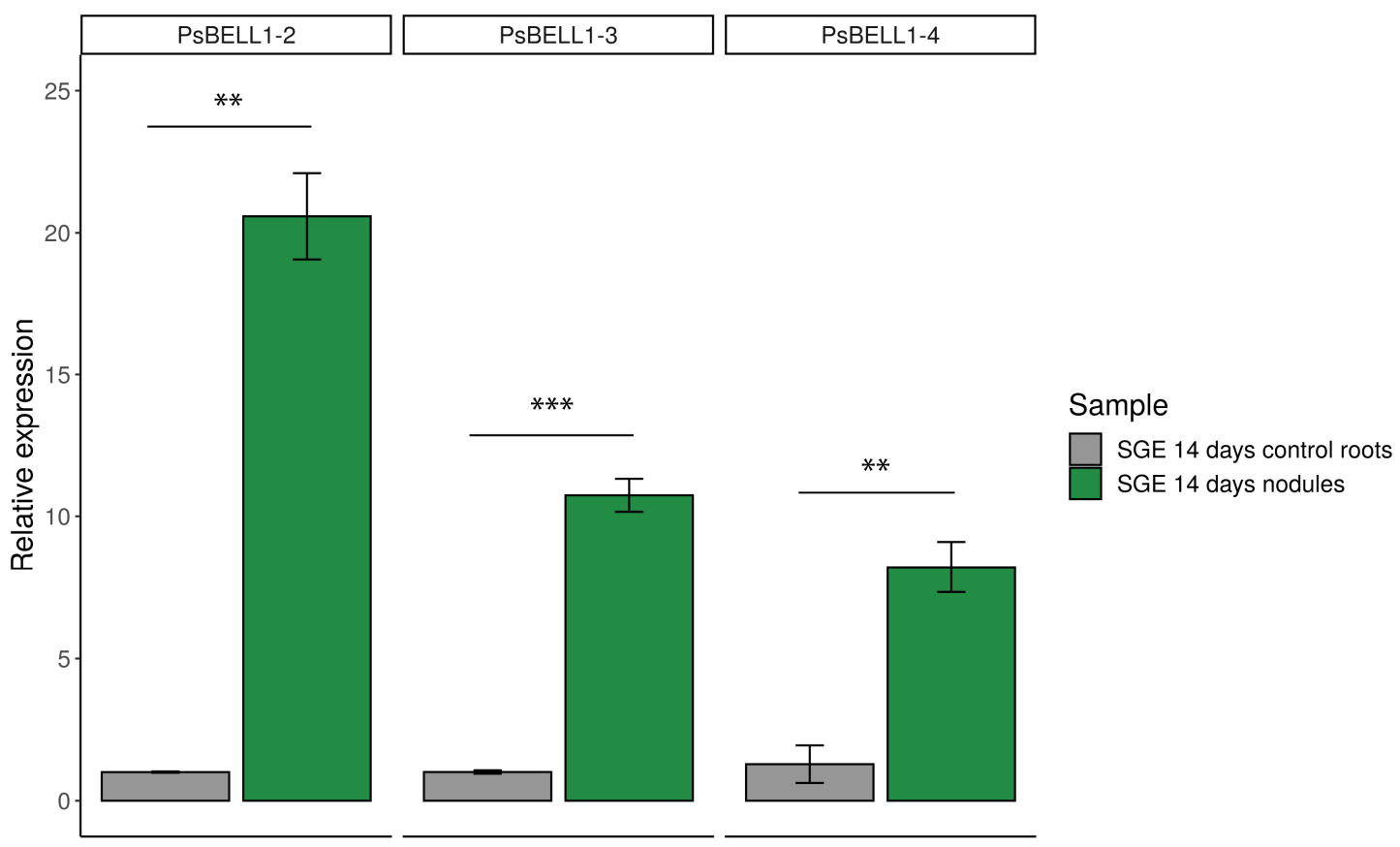

Figure 3. RT-qPCR analysis of PsBELL1-2 (Psat4g090560), PsBELL1-3 (Psat0s189g0080) and PsBELL1-4 (Psat7g031320) expression in pea nodules (SGE line). mRNA levels were normalized against Ubiquitin and values were calculated as ratios relative to non-inoculated root expression levels (control). The data of three independent biological experiments were analyzed. Bars represent the mean \pm SEM. The asterisks indicate a significant induction in nodules relative to control roots based on Student's t-test analysis $\left({ }^{* *} p<0.01,{ }^{* * *} p<0.001\right)$.

\subsection{Search for BELL Genes Regulated by NIN Transcription Factor during Symbiosis Development}

Based on data from ChIP-Seq analysis for model legume Lotus japonicus, the BELL gene (Lj4g3v2976870.1, L. japonicus genome sequence v3) was detected as a possible target of the NIN transcription factor [19]. The homologs of this gene in M. truncatula and P. sativum were determined to be MtBELL1-4 and PSBELL1-4, respectively. Since the NIN transcription factor plays a central role in nodule organogenesis and infection processes, the search for additional BELL genes that are regulated by this protein is of great interest. Here, from ChIP-Seq in L. japonicus, we extracted sequences of all available $B E L L$ genes detected as possible targets for the NIN transcription factor. BLAST analysis for screening of L. japonicus ChIP-Seq was performed using M. truncatula and P. sativum $B E L L$ gene sequences as a local database. As a result, we detected five additional BELL genes as possible targets of the NIN transcription factor in L. japonicus. To establish the relationship among these genes in different legumes, we constructed a phylogenetic tree (Figure $4 \mathrm{~A}$ ). Interestingly, four of the six detected genes were identified as homologs of genes that are highly expressed in M. truncatula- and P. sativum-inoculated roots and nodules (Figures S1-S4). The homologues of BELL1-2 and BELL1-4 genes, which increased in expression at the early stages of symbiosis development, also had the highest enrichment score, which might indicate their regulation by the NIN transcription factor at early stages of symbiosis development (Table S2). 

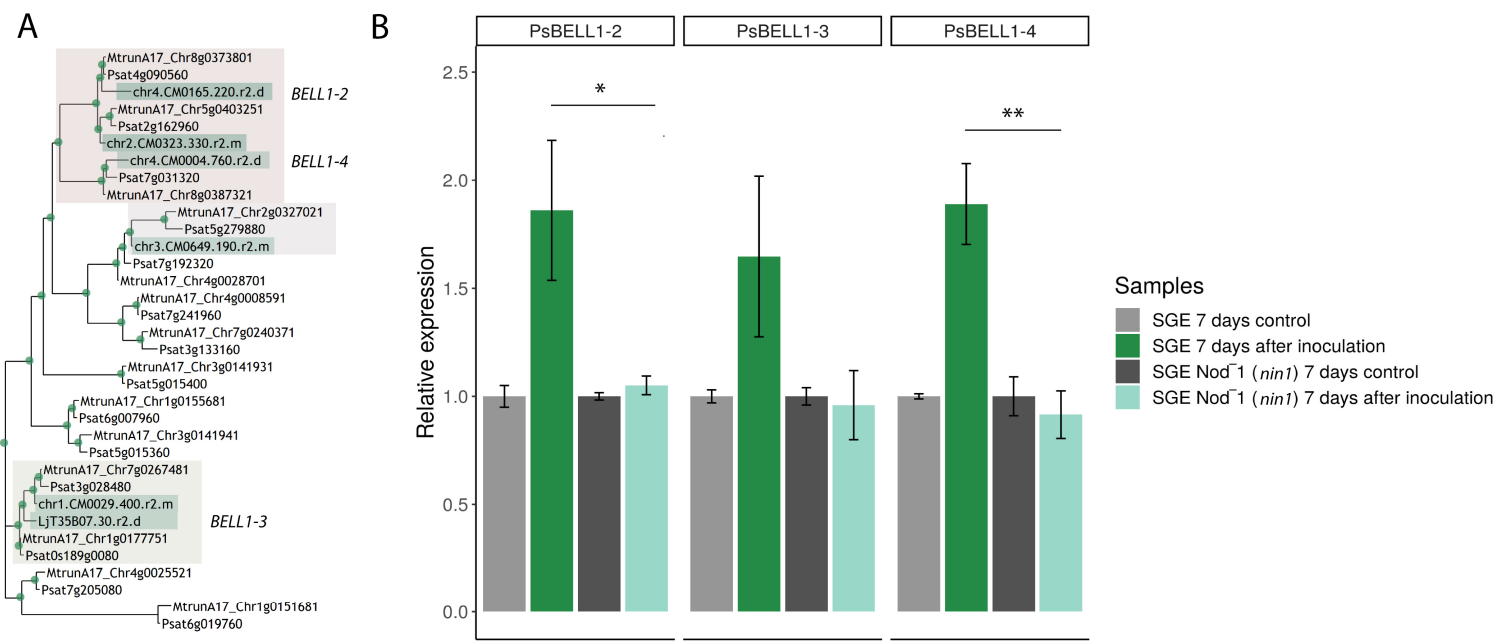

Figure 4. Phylogenetic tree constructed by the maximum-likelihood method based on amino acid sequences of Medicago truncatula and Pisum sativum BELL genes and Lotus japonicus homologous BELL genes detected in ChIP-Seq analysis. Green dots indicate support more than 70 based on 1000 bootstrap replicates (A). Expression pattern of PsBELL1-2 (Psat4g090560), PsBELL1-3 (Psat0s189g0080) and PsBELL1-4 (Psat7g031320) genes in the roots of pea SGENod ${ }^{-}-1$ (Psnin-1) mutant and wild type $P$. sativum cv. SGE (B). mRNA levels were normalized against Ubiquitin and values were calculated as ratios relative to non-inoculated root expression levels (control). The data of three independent biological experiments were analyzed. Bars represent the mean \pm SEM. The asterisks indicate statistically significant differences based on one-way analysis of variance (one-way ANOVA), followed by Tukey's post-hoc test $\left({ }^{*} p<0.05 ; * * 00.01\right)$.

\subsection{Expression of PsBELL1-2, PsBEL L1-3, and PsBELL1-4 Genes in Pea SGENod ${ }^{-}-1$ (Psnin-1) Mutant}

To evaluate the dependence of BELL gene expression on the NIN transcription factor in pea, a comparative analysis was performed between wild-type pea plants (SGE line) and the pea SGENod ${ }^{-}-1$ (Psnin-1) mutant. Whereas the expression dynamics data revealed that PsBELL1-2, PsBELL1-3, and PSBELL1-4 levels started to increase significantly at 7 dai (Figure 2), we examined whether such induction takes place in P. sativum Psnin-1 mutant at the same stage. Indeed, we did not observe an expression increase in these genes in the SGENod ${ }^{-}-1$ (Psnin-1) mutant, in contrast to the increased expression of PSBELL1-2, PSBELL1-3, and PSBELL1-4 in response to inoculation in the wild-type (Figure 4B). These data suggest that PSBELL1-2, PSBELL1-3, and PSBELL1-4 are possible targets of the NIN transcription factor in pea.

\subsection{Analysis of Interaction between PSBELL and PSKNOX Regulators during Nodulation}

Since it has been previously shown that BELL transcription factors might interact with KNOX transcription factors during the regulation of plant developmental processes [4], we suggested that BELL transcription factors could form complexes with KNOX regulators during symbiosis development. This might also be related to the regulation of some phytohormonal pathways. The significant induction of MtKNOX3/PsKNOX3, MtKNOX5/PsKNOX5, and MtKNOX9/PsKNOX9 genes was found upon nodulation in these legumes. To verify this possible interaction, we performed a more precise analysis using the two hybrid yeast system (Y2H) (Figure 5A,C). The analysis was performed for PsBELL1-2 and PsKNOX3 with PsKNOX5 and PsKNOX9 transcription factors from pea, for which their activation during symbiosis development has been previously shown [12]. As a result, we were able to detect the interaction between PsBELL1-2 and PsKNOX9 in our experiments (Figure 5). Since both regulators are also activated at the early stages of symbiosis, the possibility of complex formation between such transcription factors points towards their involvement in the regulation of similar processes at these stages. 
A

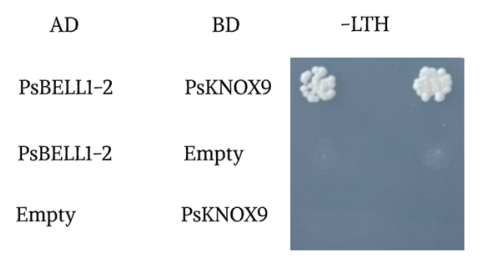

B

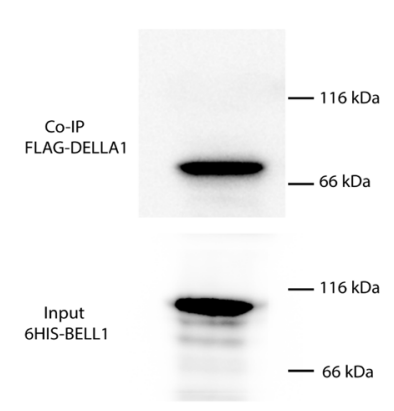

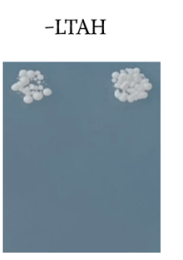

C $\mathrm{AD}$

$\begin{array}{ll}\text { PsBELLl-2 } & \text { PsDELLAl } \\ \text { PsDELLA1 } & \text { PsBELL1-2 } \\ \text { PsDELLA1 } & \text { Empty } \\ \text { Empty } & \text { PsDELLA1 } \\ \text { PsBELLl-2 } & \text { Empty }\end{array}$

Empty

PsBELLL-2
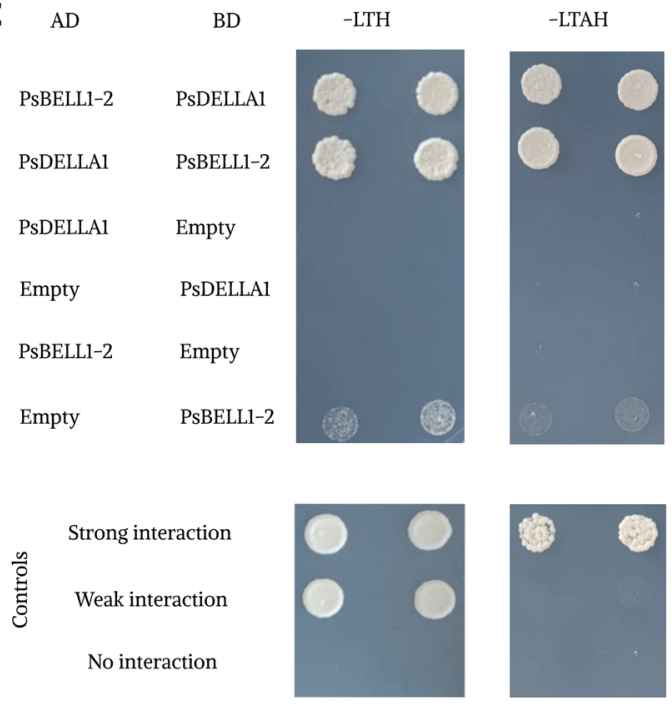

Figure 5. Analysis of the interaction between PsBELL1-2, PsKNOX9 and PsBELL1-2, PsDELLA1 using different approaches. Yeast two-hybrid assays between PsBELL1-2 and PsKNOX9 (A), PsBELL1-2 and PsDELLA1 (C). Co-immunoprecipitation (Co-IP) between PsDELLA1-FLAG and PsBELL1-2 (B). For the yeast two-hybrid assays, serial dilutions optical density at $600 \mathrm{~nm}(\mathrm{OD} 600)=0.1$ and 0.05 were used. Interaction was tested on a selective medium lacking leucine, tryptophan, and histidine (SD-LTH) and medium additionally lacking adenine (SC-LTAH). Yeast growth on indicated mediums shows the protein interaction. As controls, a few pairs of vectors (pEXP32/Krev1 and pEXP22/RalGDS-wild type, pEXP22/RalGDS-m1, and pEXP22/RalGDS-m2) suggested by the manufacturer (Thermo Fisher Scientific, Waltham, MA, USA) were used for strong, weak, and not detectable interactions.

Co-immunoprecipitation analysis was performed for PsBELL1-2 tagged with 6HIS and PsDELLA1 tagged with FLAG (Figure 5B). Proteins were synthesized in E. coli. After extraction and incubation of the proteins on ice for $1 \mathrm{~h}$, proteins were subjected to immunoprecipitation with anti-HIS resin and then analyzed on an immunoblot with anti-FLAG and anti-HIS.

\subsection{Analysis of Interaction between the Most Important Gibberellin Network Regulators, DELLA Proteins, and BELL Transcription Factors}

Since significant changes in gibberellin and cytokinin metabolism occur at the initial stages of nodulation, we studied the possible interaction between BELL transcription factors and regulators of these phytohormonal pathways. One of the most important gibberellin network regulators comprises DELLA proteins. In our previous study, we showed that PsDELLA1 and PsDELLA2 proteins had the most significant impact on pea-rhizobial symbiosis development [15]. Moreover, the expression levels of several BELL and KNOX genes were essentially decreased in the P. sativum mutants la cry-s (della1 della2). Here, we attempted to estimate the possible interaction between DELLA proteins and transcription factors from the BELL and KNOX families. Considering that the level of BELL1-2 expression was the most significant during symbiosis, we performed a more detailed analysis of PsBELL1-2 and PsDELLA1 interactions using the $\mathrm{Y} 2 \mathrm{H}$ system, as well as a co-immunoprecipitation assay. Our results reveal that PsBELL1-2 interacts with the PsDELLA1 protein based on the Y2H (Figure 5C). No interaction was observed with the empty vectors. At the same time, the co-immunoprecipitation assay to verify the PsBELL1-2 and PsDELLA1 interaction was performed (Figure 4B). Using this approach, we also confirmed the possibility of such an interaction.

\section{Discussion}

During nodule development, a complex network of transcription factors and phytohormones is involved in the regulation of the shape of this organ and its position on the root. However, 
this requires future investigations of the regulatory links between phytohormones and transcription factors, which are tightly interconnected in these processes. In this relationship, the contribution of components that are susceptible to phytohormonal action or can influence downstream components of phytohormonal pathways is crucial. Therefore, TALE proteins might be important regulators of nodule development.

It is well known that transcription factors of the TALE superfamily are important participants in processes associated with meristem maintenance and organ development [3,4]. However, specific members of this superfamily, which are involved in the regulation of definite processes, remain unknown. The role of KNOX proteins in the regulation of legume-rhizobial symbiosis was previously studied in detail. Several members of the KNOX family that belong to class II were found to be activated at the initial stages of nodule development. Therefore, it has been shown that MtKNOX3/PsKNOX3, MtKNOX5/PsKNOX5, MtKNOX9/PsKNOX9, and MtKNOX10/PsKNOX10 play an important role in nodule organogenesis and are expressed in the nodule primordium/meristem [12,13]. However, despite the fact that KNOX and BELL function in most cases as heterodimeric complexes, no data have been reported about BELLs, which are involved in this process.

Our data showed that several members of the BELL family might be involved in the control of legume-rhizobial symbiosis development. The initial stages of symbiosis are apparently associated with the activation of some BELL genes. Among them, PsBELL1-2, PsBELL1-3, and PsBELL1-4 exhibited increased expression levels in comparison with those in non-inoculated roots at the initial stages of symbiosis, as well as high expression in the nodules. Similarly, MtBELL1-2 and MtBELL1-4 homologous genes showed activation at the early symbiotic stages (1-2 dai) in $M$. truncatula, whereas their expression level was most significant in the nodules. In addition, MtBELL1-3 expression was high in M. truncatula nodules. This suggests a possible role of these genes in the regulation of nodulation.

Among these genes, PsBELL1-2 showed the most significant upregulation and will be used for future analysis in pea. The PsBELL1-2 transcription factor was shown to interact with the PsKNOX9 transcription factor, which suggests their participation in the regulation of common processes. Considering our data on the interaction between PsBELL1-2 and PsKNOX9, we suggest that BELL1-2 is of great interest for future studies. It has recently been shown that the closest homologues of PsBELL1-2 in A. thaliana (AtBLH2/AtBLH4) can influence the expression of genes encoding pectin methyl transferases (PMEs), which are important components of plant cell wall modification and are involved in the demethylesterification of homogalacturonan [20]. Interestingly, it has recently been shown that the decline of methyl esterified homogalacturonan content is specifically associated with infection thread development during nodulation [21]. Some pea mutants, SGEFix ${ }^{-}-5$ (sym33-2/ipd3) [22] and SGEFix ${ }^{-}-2$ (sym33-3/ipd3) [23], have impaired infection processes (blocking rhizobia release from infection threads) and form noneffective nodules. Previously, we found significantly decreased levels of BELL1-2 in the nodules of these mutants. It would thus be interesting to analyze the link between PsBELL1-2 activation and the level of PME gene induction in nodules of the sym33/ipd3 mutants.

It is important to note that several BELL genes were found in the ChIP-seq analysis of legume plants as possible targets of the NIN transcription factor, which is one of the most important regulators of nodule organogenesis and infection. A phylogenetic tree revealed that BELLs with the highest enrichment score also showed the highest increase in their expression level in nodules. This suggests that the expression of these BELL genes during symbiosis development might be dependent on NIN. Since the expression levels of several BELL genes are quite high in roots, temporal and spatial regulation of the expression of individual BELL genes by the NIN transcription factor might be important for their selective activation during nodulation. In contrast, only one KNOX gene was detected in the ChIP-Seq analysis of legume plants (its closest homolog in pea is PsKNOX9). Since we found that the expression dynamics of PsBELL1-2 and PSKNOX9 genes are highly similar, the possibility of their interaction is consistent with data suggesting that both of them could be regulated by NIN. However, the involvement of other KNOX regulators in the process of symbiosis development remains unclear. 
Since it was shown that BELLs are involved in the regulation of genes associated with gibberellic acid metabolism, the ability of PsBELL1-2 to interact with such components of the gibberellin signaling pathway, as is the case for PsDELLA1, is of great interest. It was shown that DELLA proteins are extremely sensitive to the presence of active gibberellins and are quickly degraded via the SCF-E3 complex in the presence of gibberellins in the environment. Considering the ability of BELL and KNOX genes to influence phytohormonal metabolism, such interactions might explain the mechanism by which TALE proteins influence gibberellin content. It is interesting to note that recent data showed the interaction between DELLA proteins and the closest homologues of PsBELL1-2 in the model plant A. thaliana, specifically AtBLH2/AtBLH4 [24]. This suggests a common tendency in the regulation of BELL transcription factors through their interaction with DELLA proteins.

\section{Materials and Methods}

\subsection{Phylogenetic Reconstruction}

A phylogenetic tree was constructed using the maximum-likelihood method with the IQ-TREE tool v.1.6.1 [25], based on amino acid sequences that were aligned using the PRANK tool v.170427 with default settings [26]. The bootstrap values were obtained from 1000 bootstrap replicates using ultrafast bootstrap [27]. Arabidopsis thaliana amino acid sequences were retrieved from the UniProt database using previously published accession numbers [3]. For the identification of Arabidopsis BELL homologues in P. sativum v1 and M. truncatula v5 genomes, the BLASTP tool v.2.6.0 (word size = 3) [16] was used with an E-value threshold of $10^{-30}$. In the phylogenetic tree for identification of L. japonicus genes from ChIP-Seq [19], we used BLASTP to detect BELL genes among the enriched genes, and the same pipeline for tree construction was used for this analysis. The $\mathrm{R}$ package ggtree was used for visualization in both cases [28]. All the sequences of BELL genes for A. thaliana, O. sativa, and the legumes can be found in the Supplementary Materials.

\subsection{RNA-Seq Quantification and Statistical Analysis}

Raw reads from the RNA-Seq project PRJNA552042 [29], including data for 24 and $48 \mathrm{~h}$ and 3, 4, 5 , and 7 days after inoculation, and mock treatment data for the same stages, were used for analysis. The Medicago truncatula genome version 5.0 was used as a reference. The reads were mapped to the genome using HISAT2 [30] tool v.2.1.0 with default parameters, and the raw counts were calculated with help of the featureCounts function from the Subread package [31]. The EdgeR package was used to calculate CPM values [32].

\subsection{Bacterial Strains and Inoculation}

Inoculation of the pea plants was performed with the Rhizobium leguminosarum biovar viciae strain 3841 [33] and CIAM1026 [34]. The bacterial liquid culture was grown at $28{ }^{\circ} \mathrm{C}$ in $\mathrm{B}^{-}$medium [35] for $24-48 \mathrm{~h}$, diluted up to the optical density at $600 \mathrm{~nm}$ (OD600) 0.5 and applied to plants one day after planting.

\subsection{Plant Material and Growth Conditions}

P. sativum L. cv. Frisson plants were used to examine the expression dynamics of BELL genes. Wild type SGE line [36] and its derived mutant SGENod ${ }^{-}-1$ (Psnin-1) [37] were also used in this study to evaluate expressions of PsBELL1-2, PsBELL1-3, and PsBELL1-4. The SGE nodules were collected at 14 days after inoculation (14 dai) and used to assess the expression level of BELL genes.

Gene expression experiments were carried out using the pea seedlings collected at different stages after inoculation. Non-inoculated roots were also collected at similar developmental stages and used as a control. To grow the seedlings, the seeds were sterilized using sulfuric acid for $5 \mathrm{~min}$, washed 3 times with water, transferred on $1 \%$ agar plates, and allowed to germinate at $23{ }^{\circ} \mathrm{C}$ in the dark. Then, 4-5-day plant seedlings were transferred into pots with vermiculite saturated with Jensen medium [38], 
grown in a growth chamber at $21^{\circ} \mathrm{C}$ and $16 \mathrm{~h}$ light $/ 8 \mathrm{~h}$ dark cycle with $60 \%$ humidity. Fragments of the main roots (responsive zone starting from 5 to $6 \mathrm{~mm}$ from the root tip) or fragments of main roots with primordia/nodules were collected at day 7 for the SGE and SGENod ${ }^{-}-1$ (Psnin-1) mutant. For the analysis of dynamics, the samples were collected at different stages after inoculation $(1,3,5,7,9,11$, and 14 dai). Fragments of non-inoculated main roots were collected at similar developmental stages. Plant material for the gene expression studies was immediately immersed in liquid nitrogen and stored in a freezer at $-80^{\circ} \mathrm{C}$.

\subsection{RNA Extraction, cDNA Synthesis, and Quantitative Reverse Transcription PCR (RT-qPCR)}

Approximately $50-100 \mathrm{mg}$ of ground plant tissue was used for RNA extraction, as previously described [39]. Around 1-2.5 $\mu$ g of total RNA was used to synthesize cDNA using $20 \mu \mathrm{L}$ RevertAid Reverse Transcriptase (Thermo Fisher Scientific, USA), and then the cDNA samples were diluted to $1: 10$. Then, $2 \mu \mathrm{L}$ of cDNA was used for quantitative PCR using Bio-Rad iQ Sybr master mix (Bio-Rad Laboratories, Hercules, CA, USA) following the manufacturer's recommendations. The CFX-96 real-time PCR detection system with C1000 thermal cycler (Bio-Rad Laboratories, USA) was used for analysis. Cycle threshold $(\mathrm{Ct})$ values were obtained using the accompanying software and data were analyzed according to the $2^{-\Delta \Delta C t}$ method [40]. All reactions were performed in triplicate and averaged. For gene expression quantification, the used primer pairs are shown in Table S3. All primers were produced by Evrogen (Moscow, Russia). The gene expression was normalized against the constitutively expressed Ubiquitin gene in pea. For temporal BELL gene expression, each replicate contained tissue of $3-4$ plants, and the experiments were repeated thrice.

\subsection{RT-qPCR Data Analysis}

One-way analysis of variance (one-way ANOVA) with post-hoc Tukey test or Student's $t$-test and relevant $R$ function were used to estimate the statistical significance. Bar plots were used to represent the relative expression values $\left(2^{-\Delta \Delta C t}\right) \pm S E M$ for three biological replicates.

\subsection{Cloning of PsDELLA1, PsKNOX3, and PsBELL1 Genes for Yeast Transformation}

The full-length coding sequences of PsDELLA1, PsKNOX9, and PsBELL1 genes were obtained by amplifying the cDNA of cv. Finale using specific PCR primer pairs flanking with attB1 and attB2 sequences or CACC sequence in the forward primer (Table S3) for subsequent cloning in pDONR221 or pENTRY vectors (Thermo Fisher Scientific, USA).

Then, these sequences were finally subcloned into the destination vectors pDEST22 (PREY) or pDEST32 (BAIT) using the LR clonase enzyme (Thermo Fisher Scientific, USA). All the verified constructs were transferred into the pJ6969-4A yeast strain.

\subsection{Yeast Two-Hybrid Assay (GAL4 Transcription Factor-Based Assay)}

The Saccharomyces cerevisiae strain pJ6969-4A [41] was transformed simultaneously with pDEST22 and pDEST32 vectors for GAL4-based selection. To transform S. cerevisiae pJ6969-4A, the protocol for preparation of chemically competent cells was used [42]. A few pairs of vectors (pEXP32/Krev1 and pEXP22/RalGDS-wild type, pEXP22/RalGDS-m1, and pEXP22/RalGDS-m2) suggested by the manufacturer as controls were used for the strong, weak, and not detectable interactions. Analyses of interactions were conducted on selective media such as synthetic complete (SC)-LT (without leucine and tryptophan), SC-LTH (without leucine, tryptophan, and histidine), and SC-LTAH (without leucine, tryptophan, adenine, and histidine).

\subsection{Protein Synthesis in E. coli and Co-Immunoprecipitation Assay}

Co-immunoprecipitation was carried out using a $\mu$ MACS kit (Miltenyi Biotec, Bergisch Gladbach, Germany) containing MicroBeads with immobilized anti-HIS antibodies. The coding sequences 
of PsBELL1-2 and PSDELLA1 genes were amplified on the cDNA and cloned in a pRSETa vector. The synthesis of proteins was performed in E. coli C41 cells. The pellets of E. coli cells were resuspended in a lysis buffer $(50 \mathrm{mM}$ tris- $\mathrm{HCl} \mathrm{pH} \mathrm{8.0,1 \%} \mathrm{Triton} \mathrm{X-100,} \mathrm{and} 150 \mathrm{mM} \mathrm{NaCl})$ and the cell lysates were obtained using sonication $(3 \times 20$ s). These lysates, containing PsBELL1-2 tagged with 6HIS and PsDELLA1 tagged with FLAG proteins, were co-incubated with the MicroBeads for $1 \mathrm{~h}$ on ice, and then they were loaded onto a MACS column placed in the magnetic field of a $\mu$ MACS separator. Next, the column with associated proteins was washed twice with the lysis buffer. After elution with a denaturing elution buffer, the precipitated proteins were analyzed using SDS-PAGE.

Supplementary Materials: The following are available online at http://www.mdpi.com/2223-7747/9/12/1808/s1, Table S1: Relationships between IDs of P. sativum, M. truncatula and A. thaliana BELL genes based on phylogenetic tree reconstruction; Table S2: IDs of L. japonicus BELL genes detected in ChIP-Seq analysis [18] as possible targets of NIN transcription factor and relevant fold enrichment score; Table S3: List of primers; Figure S1: Expression level of M. truncatula BELL genes based on re-analysis of RNA-Seq data from PRJNA552042 project using $M$. truncatula genome v.5 as a reference. Figure S2: Graphic illustration of data for P. sativum BELL genes expression in the roots and nodules based on RNA-Seq analysis; Figure S3: Graphic illustration of data for P. sativum BELL gene expression in the roots and nodules based on RNA-Seq analysis [43] using $P$. sativum genome sequence v1 as reference. Figure S4: Expression level of M. truncatula BELL genes in roots based on re-analysis of RNA-Seq data. Figure S5: Expression level of $M$. truncatula BELL genes in the different nodule zones. Data file: sequences of $B E L L$ genes for A. thaliana, O. sativa and legumes.

Author Contributions: Investigation, writing—original draft preparation, methodology, A.V.D.; investigation, E.S.R.; conceptualization, writing - review and editing, supervision, E.A.D. All authors have read and agreed to the published version of the manuscript.

Funding: This research was funded by the Russian Science Foundation (grant 16-16-10043 for genomic and transcriptomic analysis and grant 17-76-30016 for RT-qPCR, 2YH analysis and co-immunoprecipitation analysis).

Acknowledgments: The research was performed using equipment of the Core Centrum "Genomic Technologies, Proteomics and Cell Biology" in ARRIAM.

Conflicts of Interest: The authors declare they have no competing interests.

\section{References}

1. Jin, Y.; Liu, H.; Luo, D.; Yu, N.; Dong, W.; Wang, C.; Zhang, X.; Dai, H.; Yang, J.; Wang, E. DELLA proteins are common components of symbiotic rhizobial and mycorrhizal signalling pathways. Nat. Commun. 2016, 7, 12433. [CrossRef]

2. Fonouni-Farde, C.; Tan, S.; Baudin, M.; Brault, M.; Wen, J.; Mysore, K.S.; Niebel, A.; Frugier, F.; Diet, A. DELLA-mediated gibberellin signalling regulates Nod factor signalling and rhizobial infection. Nat. Commun. 2016, 7, 12636. [CrossRef] [PubMed]

3. Bürglin, T.R.; Affolter, M. Homeodomain proteins: An update. Chromosoma 2016, 125, 497-521. [CrossRef] [PubMed]

4. Hamant, O.; Pautot, V. Plant development: A TALE story. C. R. Biol. 2010, 333, 371-381. [CrossRef] [PubMed]

5. Kumar, R.; Kushalappa, K.; Godt, D.; Pidkowich, M.S.; Pastorelli, S.; Hepworth, S.R.; Haughn, G.W. The Arabidopsis BEL1-LIKE HOMEODOMAIN proteins SAW1 and SAW2 act redundantly to regulate KNOX expression spatially in leaf margins. Plant Cell 2007, 19, 2719-2735. [CrossRef] [PubMed]

6. Liu, Y.; You, S.; Taylor-Teeples, M.; Li, W.L.; Schuetz, M.; Brady, S.M.; Douglas, C.J. BEL1-LIKE HOMEODOMAIN6 and KNOTTED ARABIDOPSIS THALIANA7 interact and regulate secondary cell wall formation via repression of REVOLUTA. Plant Cell 2014, 26, 4843-4861. [CrossRef]

7. Jasinski, S.; Piazza, P.; Craft, J.; Hay, A.; Woolley, L.; Rieu, I.; Phillips, A.; Hedden, P.; Tsiantis, M. KNOX action in Arabidopsis is mediated by coordinate regulation of cytokinin and gibberellin activities. Curr. Biol. 2005, 15, 1560-1565. [CrossRef]

8. Dean, G.; Casson, S.; Lindsey, K. KNAT6 gene of Arabidopsis is expressed in roots and is required for correct lateral root formation. Plant Mol. Biol. 2004, 54, 71-84. [CrossRef]

9. Kim, J.Y.; Rim, Y.; Wang, J.; Jackson, D. A novel cell-to-cell trafficking assay indicates that the KNOX homeodomain is necessary and sufficient for intercellular protein and mRNA trafficking. Genes Dev. 2005, 19, 788-793. [CrossRef] 
10. Souček, P.; Klíma, P.; Reková, A.; Brzobohatý, B. Involvement of hormones and KNOXI genes in early Arabidopsis seedling development. J. Exp. Bot. 2007, 58, 3797-3810. [CrossRef]

11. Souček, P.; Hanáček, P.; Mazura, P.; Reinöhl, V. Interaction among BREVIPEDICELLUS, BLH6 and auxin in roots of Arabidopsis thaliana. Russ. J. Plant Physiol. 2017, 64, 386-397. [CrossRef]

12. Di Giacomo, E.; Laffont, C.; Sciarra, F.; Iannelli, M.A.; Frugier, F.; Frugis, G. KNAT3/4/5-like class 2 KNOX transcription factors are involved in Medicago truncatula symbiotic nodule organ development. New Phytol. 2017, 213, 822-837. [CrossRef] [PubMed]

13. Azarakhsh, M.; Kirienko, A.N.; Zhukov, V.A.; Lebedeva, M.A.; Dolgikh, E.A.; Lutova, L.A. KNOTTED1-LIKE HOMEOBOX 3: A new regulator of symbiotic nodule development. J. Exp. Bot. 2015, 66, 7181-7195. [CrossRef] [PubMed]

14. Azarakhsh, M.; Rumjantsev, A.; Lebedeva, M.; Lutova, L. Cytokinin biosynthesis genes expressed during nodule organogenesis are directly regulated by the KNOX3 protein in Medicago truncatula. PLoS ONE 2020, 15, e0232352. [CrossRef]

15. Dolgikh, A.V.; Kirienko, A.N.; Tikhonovich, I.A.; Foo, E.; Dolgikh, E.A. The DELLA proteins influence the expression of cytokinin biosynthesis and response genes during nodulation. Front. Plant Sci. 2019, 10, 432. [CrossRef]

16. Camacho, C.; Coulouris, G.; Avagyan, V.; Ma, N.; Papadopoulos, J.; Bealer, K.; Madden, T.L. BLAST+: Architecture and applications. BMC Bioinform. 2009, 10, 421. [CrossRef]

17. Pecrix, Y.; Staton, S.E.; Sallet, E.; Lelandais-Brière, C.; Moreau, S.; Carrère, S.; Blein, T.; Jardinaud, M.F.; Latrasse, D.; Zouine, M.; et al. Whole-genome landscape of Medicago truncatula symbiotic genes. Nat. Plants 2018, 4, 1017-1025. [CrossRef]

18. Kreplak, J.; Madoui, M.A.; Cápal, P.; Novák, P.; Labadie, K.; Aubert, G.; Bayer, P.E.; Gali, K.K.; Syme, R.A.; Main, D.; et al. A reference genome for pea provides insight into legume genome evolution. Nat. Genet. 2019, 51, 1411-1422. [CrossRef]

19. Soyano, T.; Hirakawa, H.; Sato, S.; Hayashi, M.; Kawaguchi, M. NODULE INCEPTION creates a long-distance negative feedback loop involved in homeostatic regulation of nodule organ production. Proc. Natl. Acad. Sci. USA 2014, 111, 14607-14612. [CrossRef]

20. Xu, Y.; Wang, Y.; Wang, X.; Pei, S.; Kong, Y.; Hu, R.; Zhou, G. Transcription Factors BLH2 and BLH4 Regulate Demethylesterification of Homogalacturonan in Seed Mucilage. Plant Physiol. 2020, 183, 96-111. [CrossRef]

21. Tsyganova, A.V.; Seliverstova, E.V.; Brewin, N.J.; Tsyganov, V.E. Comparative analysis of remodelling of the plant-microbe interface in Pisum sativum and Medicago truncatula symbiotic nodules. Protoplasma 2019, 256, 983-996. [CrossRef] [PubMed]

22. Tsyganova, A.V.; Ivanova, K.A.; Tsyganov, V.E. Histological and ultrastructural nodule organization of the pea (Pisum sativum) mutant SGEFix ${ }^{-}-5$ in the Sym33 gene encoding the transcription factor PsCYCLOPS/PsIPD3. Ecol. Genet. 2019, 17, 65-70. [CrossRef]

23. Tsyganov, V.E.; Morzhina, E.V.; Stefanov, S.Y.; Borisov, A.Y.; Lebsky, V.K.; Tikhonovich, I.A. The pea (Pisum sativum L.) genes sym33 and sym40 control infection thread formation and root nodule function. Mol. Gen. Genet. 1998, 259, 491-503. [CrossRef] [PubMed]

24. Altmann, M.; Altmann, S.; Rodriguez, P.A.; Weller, B.; Elorduy Vergara, L.; Palme, J.; Marín-de la Rosa, N.; Sauer, M.; Wenig, M.; Villaécija-Aguilar, J.A.; et al. Extensive signal integration by the phytohormone protein network. Nature 2020, 583, 271-276. [CrossRef]

25. Nguyen, L.T.; Schmidt, H.A.; von Haeseler, A.; Minh, B.Q. IQ-TREE: A fast and effective stochastic algorithm for estimating maximum-likelihood phylogenies. Mol. Biol. Evol. 2015, 32, 268-274. [CrossRef]

26. Löytynoja, A. Phylogeny-Aware Alignment with PRANK BT-Multiple Sequence Alignment Methods; Russell, D.J., Ed.; Humana Press: Totowa, NJ, USA, 2014; pp. 155-170. ISBN 978-1-62703-646-7.

27. Hoang, D.T.; Chernomor, O.; von Haeseler, A.; Minh, B.Q.; Vinh, L.S. UFBoot2: Improving the ultrafast bootstrap approximation. Mol. Biol. Evol. 2018, 35, 518-522. [CrossRef]

28. Yu, G.; Smith, D.K.; Zhu, H.; Guan, Y.; Lam, T.T.Y. ggtree: An r package for visualization and annotation of phylogenetic trees with their covariates and other associated data. Methods Ecol. Evol. 2017, 8, 28-36. [CrossRef]

29. Schiessl, K.; Lilley, J.; Lee, T.; Tamvakis, I.; Kohlen, W.; Bailey, P.; Thomas, A.; Luptak, J.; Karunakaran, R.; Carpenter, M.; et al. Nodule inception recruits the lateral root the lateral root developmental program for symbiotic nodule organogenesis in Medicago truncatula. Curr. Biol. 2019, 29, 3657-3668. [CrossRef] 
30. Kim, D.; Paggi, J.M.; Park, C.; Bennett, C.; Salzberg, S.L. Graph-based genome alignment and genotyping with HISAT2 and HISAT-genotype. Nat. Biotechnol. 2019, 37, 907-915. [CrossRef]

31. Liao, Y.; Smyth, G.K.; Shi, W. featureCounts: An efficient general purpose program for assigning sequence reads to genomic features. Bioinformatics 2014, 30, 923-930. [CrossRef]

32. Robinson, M.D.; McCarthy, D.J.; Smyth, G.K. edgeR: A Bioconductor package for differential expression analysis of digital gene expression data. Bioinformatics 2010, 26, 139-140. [CrossRef] [PubMed]

33. Glenn, A.R.; Poole, P.S.; Hudman, J.F. Succinate uptake by free-living and bacteroid forms of Rhizobium leguminosarum. Microbiology 1980, 119, 267-271. [CrossRef]

34. Safronova, V.I.; Novikova, N.I. Comparison of two methods for root nodule bacteria preservation: Lyophilization and liquid nitrogen freezing. J. Microbiol. Methods 1996, 24, 231-237. [CrossRef]

35. Van Brussel, A.; Planqué, K.; Quispel, A. The wall of Rhizobium leguminosarum in bacteroid and free-living forms. J. Gen. Microbiol. 1977, 101, 51-56. [CrossRef]

36. Kosterin, O.E.; Rozov, S.M. Mapping of the new mutation blb and the problem of integrity of linkage group I. Pisum Genet. 1993, 25, 27-31.

37. Tsyganov, V.E.; Voroshilova, V.A.; Kukalev, A.S.; Azarova, T.S.; Yakobi, L.M.; Borisov, A.Y.; Tikhonovich, I.A. Pisum sativum L. genes sym14 and sym 35 control infection thread growth initiation during the development of symbiotic nodules. Russ. J. Genet. 1999, 35, 284-291.

38. Van Brussel, A.A.N.; Tak, T.; Wetselaar, A.; Pees, E.; Wijffelman, C.A. Small leguminosae as test plants for nodulation of Rhizobium leguminosarum and other rhizobia and agrobacteria harbouring a leguminosarum sym-plasmid. Plant Sci. Lett. 1982, 27, 317-325. [CrossRef]

39. Dolgikh, E.A.; Kusakin, P.G.; Kitaeva, A.B.; Tsyganova, A.V.; Kirienko, A.N.; Leppyanen, I.V.; Dolgikh, A.V.; Ilina, E.L.; Demchenko, K.N.; Tikhonovich, I.A.; et al. Mutational analysis indicates that abnormalities in rhizobial infection and subsequent plant cell and bacteroid differentiation in pea (Pisum sativum) nodules coincide with abnormal cytokinin responses and localization. Ann. Bot. 2020, 125, 905-923. [CrossRef]

40. Livak, K.J.; Schmittgen, T.D. Analysis of relative gene expression data using real-time quantitative PCR and the 2- $\Delta \Delta$ CT Method. Methods 2001, 25, 402-408. [CrossRef]

41. James, P.; Halladay, J.; Craig, E.A. Genomic libraries and a host strain designed for highly efficient two-hybrid selection in yeast. Genetics 1996, 144, 1425-1436.

42. Gietz, R.D.; Schiestl, R.H. Frozen competent yeast cells that can be transformed with high efficiency using the LiAc/SS carrier DNA/PEG method. Nat. Protoc. 2007, 2, 1-4. [CrossRef] [PubMed]

43. Alves-Carvalho, S.; Aubert, G.; Carrère, S.; Cruaud, C.; Brochot, A.L.; Jacquin, F.; Klein, A.; Martin, C.; Boucherot, K.; Kreplak, J.; et al. Full length de novo assembly of RNA-seq data in pea (Pisum sativum L.) provides a gene expression atlas and gives insights into root nodulation in this species. Plant J. 2015, 84, 1-19. [CrossRef] [PubMed]

Publisher's Note: MDPI stays neutral with regard to jurisdictional claims in published maps and institutional affiliations.

(C) 2020 by the authors. Licensee MDPI, Basel, Switzerland. This article is an open access article distributed under the terms and conditions of the Creative Commons Attribution (CC BY) license (http://creativecommons.org/licenses/by/4.0/). 